\title{
Status Monitoring and Fault Analysis of Aircraft Control System
}

\author{
Ge Yunpeng, Wang Jiaming, Liu Shuo, Liu Yuhang, Huang Xiaoqing
}

Cetc Wuhu Diamond Aircraft Manufacture Co., Ltd., Wuhu, China

Keywords: Aircraft control system, System monitoring, Fault handling

\begin{abstract}
Flight data recording (FDR) system as an important data acquisition and processing system, the aircraft will normally collected in plane state monitoring system of data records to the aircraft airborne equipment CARDS, in order to achieve the aircraft condition monitoring and fault diagnosis, in order to reduce the probability of unplanned maintenance time, increase the aircraft flight safety and efficiency, based on the general structure and operation principle of control system, focus on the analysis of the flaps in control system running state monitoring and fault problem analysis, in order to improve the stability of the aircraft control system, plays a very important practical value in the cost of maintenance.
\end{abstract}

\section{Introduction}

Aircraft control system is the key to the main control aircraft flight safety system, it can ensure the aircraft in normal flight condition, so it is very important one of airborne electronic equipment, at the same time, the stability of the control system with aircraft flight safety are closely linked, now because of the control system is out of control, the cause of the accident events, embodied in resulting in failure of aircraft cannot manipulate or accident signs, such as flap failure occurs in a plane operating system of the probability is bigger, flap system is important in the flight control system, used in the plane or in the process of land to increase the lift, It can also be regarded as a lifting device. The existence of flaps can ensure that the aircraft can take off and land at a lower speed, so it is of great practical value to study it.

\section{Overview of Aircraft Control System}

Aircraft as early as in the system is mainly used for the control instruction, which drive actuator motion device for the aircraft aerodynamic shape, flight attitude control, control the aircraft rudder surface can be achieved for aircraft around the horizontal axis and vertical axis and vertical axis of rotation, which control the aircraft flight status, and the system can ensure the aircraft in flight in the early stage of maintain posture, aircraft wings and tail section is divided into two types of control system, specific flight control as the primary flight control system and auxiliary system, the control plane is the movement of transverse and longitudinal axis and vertical axis, the latter mainly for adjustment, Is to effectively improve the aircraft operating system handling and climbing performance.

Flap system of aircraft control system is the important composition of building, by the increase of the wing area to protect the takeoff and landing of aircraft at low speed still have enough lift, to ensure that the plane of resistance, speed and fuel consumption to achieve the best condition, then improve the take-off and landing performance, as rising device in seam plane fly wing system is divided into two parts namely the lateral device flap and medial flap. 


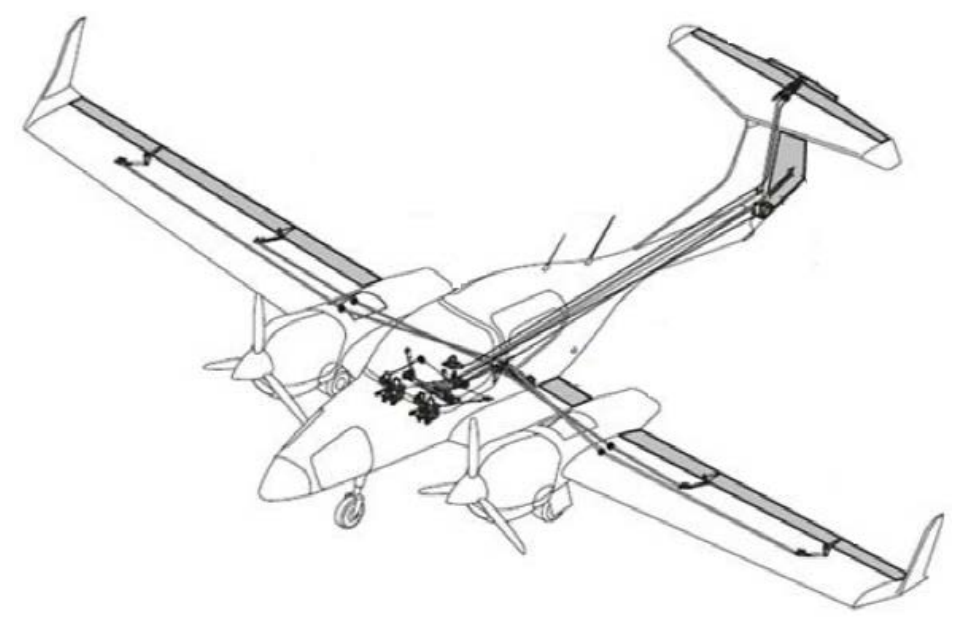

Fig.1 Schematic Structure of Aircraft Control System

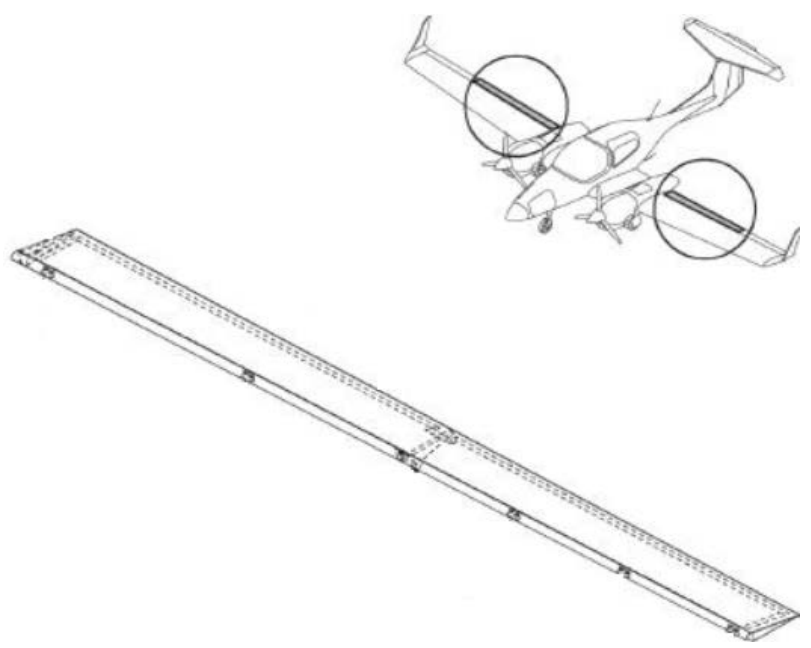

Fig.2 Outer Flaps Illustrate Structure
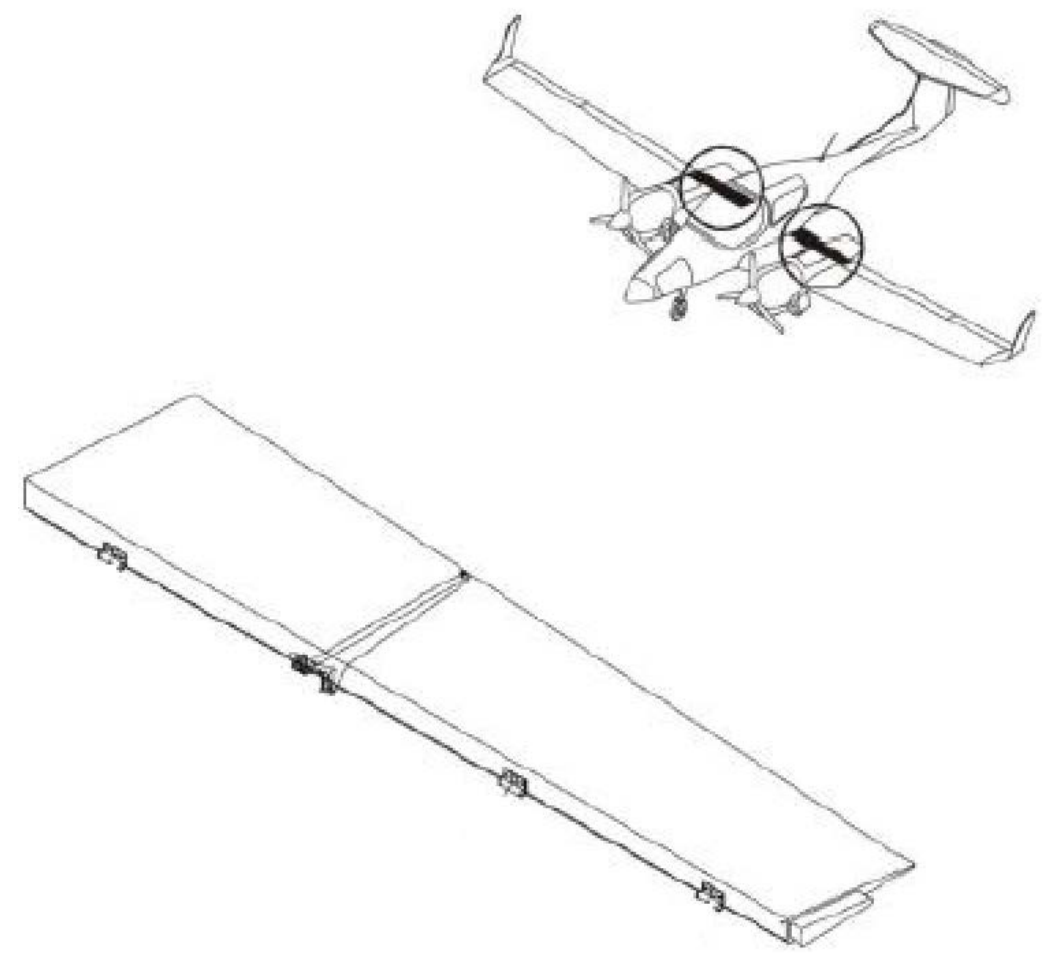

Fig.3 Inner Flaps Show the Structure 


\section{Aircraft Flight Data Acquisition and Control System Monitoring}

Aircraft in flight data mainly includes the aircraft flight mission when the motion state and working state of the corresponding parameters, the data relating to sort is more, it is mainly used for after the accident investigation, analysis, and the quality monitoring and aircraft maintenance, flight vehicle data acquisition system can through the sensor, signal according to certain order and time for gathering and transferring his recorder to record data, in order to identify the fault plane flight, after by lighting and alarm devices such as feedback to drive its purpose is to more precise complex data processing, different state monitoring of aircraft, Fault identification and fault model construction can be carried out as early as possible, and correct flight data can provide foundation for aircraft operation after decoding.

For plane detection systems need to choose the appropriate testing, so that better reflects the current plane running state, there are many means the choice of the amount for the moment the plane monitoring including direct monitoring of aircraft parameters, including the plane deflection sensor, to monitor if the sensor Angle $45^{\circ}$, can directly determine change system failure occurs, the data also can compare monitoring, such as aircraft flap Angle difference shall not be higher than $1^{\circ}$, therefore when testing, comparing the need of aircraft flap about parameters calculated by the difference to judge the fault, and the characteristics of extraction of plane numerical, Such as through the extraction of the aircraft flap Angle numerical can determine the flaps open time consumed, and the third way to extract the characteristic value of strong general features, therefore in the practical application of the combination of the characteristic flight parameters and definition, flying by extracting the characteristic parameters of way found to be the characteristics of monitoring, and obtain flight state and fault.

\section{Aircraft Flap System Analysis and Failure Monitoring}

In aircraft FDR recorded a lot about the aircraft flight parameters, usually an aircraft's record information system is mainly including aircraft attitude information, audio information, the rudder balancing and steering column information, such as its record for flap system including the content of the switch position information, the flap flap supervision information and some instructions, etc., which need to pay attention to the monitoring and control system is switch the location of the flap, flap location parameters. Only $13.04 \%$ of the data used to identify flaps failure of aircraft operating system by using FDR data cannot be analyzed, which shows that it has a very high coverage rate. Common faults include deflection sensor fault and flaps Angle anomaly.

Trend due to aircraft control system performance is relatively hidden, through the way of fault prediction can effectively find the hidden changes, is advantageous for the disposal of the early yuan, so the flight fault elimination, to improve the stability of aircraft operation, therefore FDR data extract on the eigenvalues of the aircraft fault trends, using Iaero software with ARIM model can implement for aircraft fault prediction, thus and sentenced to a period of time in the future aircraft may change trend, or is a time when the aircraft will fail.

\section{Conclusion}

In this article use plane FDR data to realize the monitoring and fault analysis of aircraft control system, the first is the structure of the aircraft control system is introduced briefly, the aircraft flight data acquisition and control system of monitoring, especially compared with the aircraft flap system failure rate is higher, therefore to aircraft flap as the research object in the control system on the system analysis and fault monitoring are summarized, its purpose is to understand how the aircraft control system is to feature extraction and analysis of data.

\section{References}

[1] Wu zhentao, li xue-ren, du jun, ding chao. Fault assessment method of aircraft control system based on flight data [J]. Computer measurement and control, 2019, 27(07):275-279. 
[2] Li hongliang, zhang wei-hui, Lin ji-feng, zhang ning. Application of mode conversion in aircraft control system test [J]. Engineering and test, 2019, 59(01):42-44+84.

[3] Jiang Zhunan. Study on health Assessment and Fault Diagnosis of aircraft control System [D]. Nanjing University of Aeronautics and Astronautics, 2018.

[4] Xie xihua, Chen zhiwei, ouyang xing, wang xiaofei, wang xiaoling. Research on improved RSSR mechanism in aileron control system of light aircraft [J]. Progress in aeronautical engineering, 2017,8(02):213-218.

[5] Huang Guolong, ZHANG Liguo. Parametric Design and Simulation Research of Longitudinal Control System of Light Aircraft [J]. China High-tech Enterprise,2014(33):33-35. 